\title{
Bottom and Charm Production at LHC and RHIC
}

\author{
Jens Ole Schmitt, Gouranga C. Nayak, Horst Stöcker and Walter Greiner \\ Institut für Theoretische Physik, J. W. Goethe-Universität, 60054 Frankfurt am Main, Germany
}

\begin{abstract}
We study $b \bar{b}$ and $c \bar{c}$ production and the influence of nuclear shadowing at LHC and RHIC energies. We find a significant reduction in the production cross section of both charm and bottom at RHIC and LHC. Bound states such as $\Upsilon$ and $J / \Psi$ are suppressed by this reduction in the charm production cross sections. Therefore, $J / \Psi$ suppression may not be useful as a signature for the quark gluon plasma.
\end{abstract}

PACS: 12.38.Mh, 25.75.-q, 24.85.+p, 14.65.Dw 
As lattice QCD results indicated, hadronic matter probably undergoes a phase transition into a deconfined quark-gluon plasma (QGP) phase, provided a temperature about 200 $\mathrm{MeV}$ or an energy density $\sim 2 \mathrm{GeV} / \mathrm{fm}^{3}$ is reached [1]. High energy heavy-ion colliders at RHIC (Au-Au at $\sqrt{s}=200 \mathrm{GeV}$ ) and $\mathrm{LHC}(\mathrm{Pb}-\mathrm{Pb}$ at $\sqrt{s}=5.5 \mathrm{TeV})$ will provide the best opportunity to study the formation and evolution of quark-gluon plasma [2]. As QGP exists only for a very short time (several fermi) in a small volume $\left(\sim 100 \mathrm{fm}^{3}\right)$, a direct detection of this state of matter is not possible. Thus various indirect signatures have to be used for its detection. The prominent ones among the suggested signatures are $J / \Psi$ suppression [3], strangeness enhancement [4, 5], dilepton and photon production [6-9]. Although these signatures are proposed, it is not easy to make a concrete calculation of them for these experiments. Many uncertainties, both theoretical and experimental, exist which make it very difficult to claim the existence of the quark-gluon plasma based on the measurement of these signatures. At the present experiment at SPS most of the signatures can be explained by alternative methods without assuming the existence of quark-gluon plasma. In this paper we will study the impact of such non-QGP sources on the production of $J / \Psi$ at RHIC and LHC energies.

We mention here that due to the presence of nuclear, quark-gluon and hadronic medium a correct computation of $J / \Psi$ suppression in ultra relativistic heavy-ion collisions (URHIC) becomes extremely difficult. We highlight here the major uncertainties associated with the calculation of $J / \Psi$ suppression. First of all one has to understand the production of $J / \Psi$ in nuclear collisions before addressing its suppressions. The main uncertainty lies in the accurate determination of the non-perturbative matrix element for $J / \Psi$ production from the $C \bar{C}$ pair. Before a decade ago one used to use the colour singlet model for $J / \Psi$ production at high energy hadronic collisions where it is assumed that the $C \bar{C}$ is produced directly as a colour singlet state which forms the $J / \Psi$ bound state [10]. However, the colour singlet model failed to describe the high momenta $J / \Psi$ production data at the CDF experiment [11]. An effective QCD formalism based on non-relativistic quantum chromodynamics was developed where one assumes that the $C \bar{C}$ is formed as a colour octet state which finally emits a soft gluon to form a colour singlet $J / \Psi$ [12]. This model (known as colour octet model) is very successful in explaining the $J / \Psi$ production data at the CDF experiment at Fermi Lab [11]. In this model the non-perturbative matrix element for $J / \Psi$ formation from $C \bar{C}$ is determined from the experiment. However, it is not at all clear how to obtain this nonperturbative matrix element for high energy nucleus-nucleus collisions. The complication arises due to the presence of a nuclear/quark-gluon medium. The second uncertainty lies in the accurate determination of the nuclear partonic structure function used to compute $C \bar{C}$. Once these two issues are solved then one can compute $J / \Psi$ production in high energy heavy-ion collisions. We will address here how significantly the heavy quark production cross section is changed when modified nuclear partonic structure functions are taken into account. This will have a direct impact on the quarkonium production cross section in high energy heavy-ion collisions at RHIC and LHC.

Before proceeding to our calculation we briefly mention some of the other effects which must be considered for a correct calculation of $J / \Psi$ suppression. First of all we mention that the space-time evolution of quark-gluon plasma in ultra relativistic heavy-ion collisions is described in various stages, namely; 1) Pre-equilibrium, 2) Equilibrium and 3) Hadronization. Once $C \bar{C}$ is formed, it travels in pre-equilibrium stage of the quark-gluon plasma. The 
charm quarks scatter with the light quarks and gluons in the medium and hence change the energy, momentum and separation of the $C \bar{C}$ pairs. It is not easy to keep track of all the interactions a charm quark encounters during its propagation in the non-equilibrium medium. This is because a correct description of non-equilibrium space-time evolution of the quarks and gluons formed in nuclear collisions is not solved accurately. On the other hand, if a $J / \Psi$ is formed, then its interaction with quarks and gluons in non-equilibrium medium has also to be taken into account. In the equilibrium stage one can argue that they are screened. But a real dynamical screening argument has to be given. The screening argument given in [3] is valid for a static plasma as calculations were done in lattice QCD which assumes a static temperature. In heavy-ion collisions the temperature is local and space-time dependent and a screening calculation involving local space-time dependent temperature is not available. The $J / \Psi$ also interacts with the hadrons in the hadronic phase 13, 14. Hence all these effects have to be considered in the study of $J / \Psi$ suppression at RHIC and LHC.

In this paper we address one of the above issues, i.e. we compute heavy-quark production at RHIC and LHC by taking modifications of the nuclear partonic distribution functions into account. The modification of nuclear parton distribution functions (i.e, $F_{2}^{A}\left(x, Q^{2}\right) \neq$ $A F_{2}^{N}\left(x, Q^{2}\right)$ and $\left.G^{A}\left(x, Q^{2}\right) \neq A G^{N}\left(x, Q^{2}\right)\right)$ has been observed in various experiments [15]. Here $F_{2}^{A(N)}\left(x, Q^{2}\right)$ and $G^{A(N)}\left(x, Q^{2}\right)$ are the quark and gluon structure functions of the nucleus (nucleon), $x$ is the momentum fraction of the parton, and $Q^{2}$ is the momentum transfer in deep inelastic lepton nucleus (nucleon) scattering. We take the modified parton distribution functions from a previous study [16], where it has been shown that there is a strong shadowing of the parton distribution functions at low $x$. The idea of parton fusion was first formulated in [17] and later proven in [18] to appear when the total transverse size 1/Q of the partons in a nucleon becomes larger than the proton radius to yield a transverse overlapping within a unit of rapidity $x G(x) \geq Q^{2} R^{2}$. The fusion correction in the free nucleon turns out to be significant only for unusually small values of $x$ and $Q^{2}$. However, in the case of heavy nuclei of $\mathrm{A} \sim 200$, the parton recombination is strongly increased (see [16, 19,20]). This strong shadowing is expected to reduce the heavy quark production significantly. We incorporate this feature of gluon shadowing in the calculation of $C \bar{C}$ production at RHIC and LHC heavy-ion collisions.

We use perturbative QCD methods to compute charm and bottom production at RHIC and LHC nuclear collisions. As we are interested in comparing the results of heavy-quark production using unshadowed and shadowed parton distributions we will not do a nextto-leading order calculation for $Q \bar{Q}$ production. For our purpose we consider the leading order gluon fusion process $g\left(p_{1}\right)+g\left(p_{2}\right) \rightarrow Q\left(p_{3}\right)+\bar{Q}\left(p_{4}\right)$ for heavy quark-antiquark pair production. For simplicity a $\mathrm{K}$ factor $\sim 2$ can be multiplied with this leading order cross section to consider higher order processes [21]. The gluon fusion process is dominant at high energy hadron-hadron collisions [22]. The squared amplitude for the above leading order process is given by [23]:

$$
\begin{array}{r}
\sum\left|\mathcal{M}_{g g \rightarrow Q \bar{Q}}\right|^{2}=\pi^{2} \alpha^{2}\left(Q^{2}\right)\left[\frac{12}{s^{2}}\left(M^{2}-t\right)\left(M^{2}-u\right)\right. \\
+\frac{8}{3} \frac{\left(M^{2}-t\right)\left(M^{2}-u\right)-2 M^{2}\left(M^{2}+t\right)}{\left(M^{2}-t\right)^{2}}+\frac{8}{3} \frac{\left(M^{2}-t\right)\left(M^{2}-u\right)-2 M^{2}\left(M^{2}+u\right)}{\left(M^{2}-u\right)^{2}} \\
\left.-\frac{2 M^{2}\left(s-4 M^{2}\right)}{3\left(M^{2}-t\right)\left(M^{2}-u\right)}-6 \frac{\left(M^{2}-t\right)\left(M^{2}-u\right)+M^{2}(u-t)}{s\left(M^{2}-t\right)}-6 \frac{\left(M^{2}-t\right)\left(M^{2}-u\right)+M^{2}(t-u)}{s\left(M^{2}-u\right)}\right],
\end{array}
$$


where M is the mass of the heavy quark $Q$. The Mandelstam variables $\hat{s}, \hat{t}, \hat{u}$ are defined by

$$
\hat{s}=\left(p_{1}+p_{2}\right)^{2}, \hat{t}=\left(p_{1}-p_{3}\right)^{2}, \hat{u}=\left(p_{1}-p_{4}\right)^{2}
$$

with the four-momenta $p_{1}, p_{2}$ and $p_{3}, p_{4}$ of the incident and the scattered particles respectively. Using the amplitude for the $2 \rightarrow 2$ process, the partonic cross section can be written as

$$
\hat{\sigma}(\hat{s})=\int d t \frac{d \sigma}{d t}=\frac{1}{16 \pi s^{2}} \int_{M^{2}-s\left(1+\sqrt{1-4 M^{2} / s}\right) / 2}^{M^{2}-s\left(1-\sqrt{1-4 M^{2} / s} / 2\right.} d t \sum|\mathcal{M}|^{2} .
$$

Assuming $Q^{2}=\hat{s}$ [23], the $\alpha\left(Q^{2}\right)$ can be taken out of the $\hat{t}$ integration to find

$$
\hat{\sigma}_{g g \rightarrow Q \bar{Q}}(\hat{s})=\frac{\pi \alpha^{2}(\hat{s})}{3 \hat{s}}\left[-\left(7+\frac{31 M^{2}}{\hat{s}}\right) \frac{1}{4} \chi+\left(1+\frac{4 M^{2}}{\hat{s}}+\frac{M^{4}}{\hat{s}^{2}}\right) \log \frac{1+\chi}{1-\chi}\right]
$$

with

$$
\chi=\sqrt{1-\frac{4 M^{2}}{\hat{s}}} .
$$

This cross section in the partonic level can be used to compute the $Q \bar{Q}$ production cross section in hadronic collisions by using the parton distribution functions inside the hadron. The total cross section for $Q \bar{Q}$ production per nucleon in AA collision is:

$$
\sigma(s)_{Q \bar{Q}}=\int_{4 M^{2} / s}^{1} d x_{1} \int_{4 M^{2} /\left(s x_{1}\right)}^{1} d x_{2} g_{g}^{A}\left(x_{1}, Q^{2}\right) g_{g}^{A}\left(x_{2}, Q^{2}\right) \hat{\sigma}_{g g \rightarrow Q \bar{Q}}(\hat{s}),
$$

where $\hat{s}=x_{1} x_{2} s$, and $g^{A}\left(x, Q^{2}\right)$ is the gluon structure function of a bound nucleon inside the nucleus $A$. On these equations nuclear shadowing effects in $g^{A}\left(x, Q^{2}\right)$ will have an influence, as the nuclear structure functions are reduced in comparison to free nucleon structure functions at low $x$. We use the shadowing version of parton distribution functions in a bound nucleon from ref. [16] (with A = 208) together with the GRV98 set of gluon distribution functions for a free nucleon [24]. The strong coupling constant $\alpha(\hat{s})$ used in the above equation is also taken from [24].

We mention here that our calculation is based on primary hard collisions of partons. These primary hard collisions also produce a huge number of jets and minijets at RHIC and LHC energies. Unlike in pp collisions these minijets suffer from secondary collisions because they are large in number. Secondary collisions among these primarily produced minijets bring the system towards equilibrium. The secondary collisions among these minijets can also produce additional $C \bar{C}$ pairs. However, it should be checked whether this heavy quark production is small or large due to their large masses. It can be argued that the secondary charm production is very small for an equilibrium quark-gluon plasma because the production rate behaves like $\sim e^{-M / T}$. However, in the early pre-equilibrium stage of the quark-gluon plasma, the average energy carried by a parton is large and hence their secondary collisions can produce significant secondary $C \bar{C}$ pairs. One needs a real calculation by using non-equilibrium distribution of the minijet plasma at RHIC and LHC to see whether the secondary $C \bar{C}$ pair production becomes larger/smaller than the primary 
charm production. The secondary charm production calculation is very important because a huge production of secondary charm may suggest an enhancement of $J / \Psi$ at RHIC and LHC rather than its suppression. In any case, the shadowing will also decrease the minijet production [16]. In such situations the minijet temperature will also be reduced which in turn will decrease secondary charm production. Hence as far as shadowing of initial parton distributions is concerned, it decreases both primary and secondary charm productions. In the following we will present results of the cross section for heavy quark-antiquark pair production from primary partonic collisions at different center of mass energies to observe the effect of shadowing on quarkonium production.

In Fig. 1 we present the cross section (in mb) for heavy quark/anti-quark pair production at RHIC energy as a function of the heavy-quark mass. The dotted line is obtained by using no-shadowing gluon distribution functions, i.e, we have used $g^{A}\left(x, Q^{2}\right)=g^{N}\left(x, Q^{2}\right)$, with $g^{N}\left(x, Q^{2}\right)$ taken from the GRV98 parametrization [24] (Note that $g^{A}\left(x, Q^{2}\right)$ used in Eq. (四) is the distribution function of a bound nucleon inside a nucleus A). It can be mentioned that our results are with respect to the nucleon-nucleon scattering in nuclear collisions. To estimate the total cross section in nucleus-nucleus collisions, one has to multiply our results by $A^{2}$. The solid line is obtained by using strong shadowing of gluons at high energy [16]. It can be seen that the shadowing in gluon distribution function decreases the heavy quark production cross section significantly. The reduction of charm quark production cross section at RHIC and LHC is interesting because the suppression of $J / \Psi$ may be due to the shadowing of the initial gluon distribution functions inside the nucleus rather than existence of QGP. However, before making any conclusions, one has to compute $J / \Psi$ production by using a suitable model for hadronization. In addition to this, one has to compute secondary charm quarks which are produced from the rescattering of minijets being formed in high energy heavy-ion collisions. We have plotted the ratio of heavy-quark production cross sections with and without shadowing in the same figure. This is represented by the dashed line. Our results cover both charm and bottom quark production at RHIC. In future RHIC will provide an opportunity to measure open charm production.

In Fig. 2 we present the cross section for heavy quark/anti-quark pair production at LHC energy as a function of the heavy-quark mass. We present our results up to $M_{Q} \sim 200 \mathrm{GeV}$ which covers charm, bottom and top quark production at LHC. Dotted, dashed and solid lines correspond to the same situation as in Fig.1. The difference in charm and bottom quark production at LHC is found to be much larger than that at RHIC. It can be seen from the figure that the shadowing effect on charm production is much larger than that on bottom production. The ratio is close to 1 for the production of the top quark.

In Fig. 3 we present the $C \bar{C}$ production cross section as a function of $\sqrt{s}$. The dotted line is for the case without shadowing, and the solid line is with shadowing of gluons. It can be seen that the $C \bar{C}$ production cross section at RHIC for both cases is not much different. However, the difference becomes quite large when the calculation is repeated for very high energy nuclear collisions. At LHC energy the reduction in the cross section is quite large, about a factor of 10 . The same is true for $B \bar{B}$ production at different energies, see Fig.4. We mention here that our results are obtained from the leading order partonic scattering processes. Our results can be multiplied by a $\mathrm{K}$ factor $(\sim 2)$ to take higher order processes into account.

To summarize, we have computed heavy quark/anti-quark pair production cross sections 
per nucleon-nucleon collision in RHIC and LHC heavy-ion collisions with shadowing of parton structure functions taken into account. It is shown that the shadowing of parton distribution functions significantly decreases the production cross section of heavy quarks at LHC. At RHIC energy this difference is not very large but still significant. One can obtain the total charm production at RHIC and LHC from these cross sections via the relation $N=T(0) \sigma$, where the Glauber geometrical factor $T(0) \sim \frac{A^{2}}{\pi R_{A}^{2}}$ for the impact parameter $\mathrm{b}=0 \mathrm{fm}$. This geometrical factor represents the total number of binary nucleon-nucleon collisions in central AA collisions, which behaves like $A^{4 / 3}$ 25]. Although the difference of the $b \bar{b}$ and $c \bar{c}$ cross sections for shadowing and no shadowing at RHIC is not large, there is a significant difference in the total number of charm and bottom quarks for the two versions of shadowing. This has a direct impact on quarkonium and open charm production at RHIC. We suppose that a reduction of the charm quark cross section due to shadowing is very significant because $J / \Psi$ suppression might occur due to the depletion of the initial gluon distribution functions rather than due to the existence of a quark-gluon plasma or due to the presence of hadronic medium.

\section{ACKNOWLEDGMENTS}

We thank Lars Gerland, Nils Hammon and Chung-Wen Kao for discussions. G. C. N. acknowledges the financial support from Alexander von Humboldt Foundation. 


\section{REFERENCES}

[1] See e.g L. McLerran and B. Svetitsky, Phys. rev. D24 (1981) 450; L. McLerran, Phys. Rev. D36 (1987) 3291; R.V. Gavai, in: Quantum Fields on the Computer, ed. M. Creutz, World Scientific, Singapore 1992, p. 51; F. Karsch and E. Laermann, Rep. Prog. Phys. 56 (1993) 1347; M. Oevers, F. Karsch, E. Laermann, R. Schmidt in: Proc. of Lat. 1997 Nucl. Phys. Proc. Suppl. 63 (1998) 394.

[2] Proceedings of The $14^{\text {th }}$ International Conference on Ultrarelativistic Nucleus-nucleus Collisions (Quark Matter '99), Torino, Italy, 10-15 May 1999, Nucl. Phys. A 661 (1999).

[3] T. Matsui and H. Satz, Phys. Lett. B 178 (1986) 416.

[4] J. Rafelski and B. Mueller, Phys. Rev. Lett. 48 (1981) 1066.

[5] R. Baier and R. Rückl, Z. Phys. C 19 (1983) 251; R. Gastmans, W. Troost and T. T. Wu, Nucl. Phys. B 291 (1987) 731.

[6] M.T. Strickland, Phys. Lett. B 331 (1994) 245.

[7] E.V. Shuryak and L- Xiong, Phys. Rev. Lett. 70 (1993) 2274.

[8] Jan-e Alam, Sibaji Raha and Bikash Sinha, Phys. Rep. 273 (1996) 243.

[9] Gouranga C. Nayak, Phys. Lett. B 442 (1995) 427.

[10] E. L. Berger and D. Jones, Phys. Rev. D23 (1981) 1521; R. Baier and R. Ruckl, Phys. Lett. B102 (1981).

[11] F. Abe et al. (CDF), Phys. Rev. Lett. 69 (1992) 3704, ibid. 79 (1997) 572.

[12] G. T. Bodwin, E. Braaten and G. P. Lepage, Phys. Rev. D51 (1995) 1125; erratum ibid. D55 (1997) 5853.

[13] C. Gerschel and J. Hufner, Z.Phys. C 56 (1992) 171.

[14] Gouranga C. Nayak, JHEP 9802 (1998) 005.

[15] See, e.g. J.J. Aubert et al. (EMC), Nucl. Phys. B 293 (1987) 740; A.C. Benvenuti et al. (BCDMS) Phys. Let. B 189 (1987) 483; P. Amaudruz et al. (NMC) Z. Phys. C 53 (1992) 73; C. Benvenuti et al. (CDMS)Z. Phys.C 63 (1994) 29; B. Adeva et al. (SMC) Phys. Let. B 320 (1994) 400.

[16] N. Hammon, H. Stöcker and W. Greiner, Phys. Lett. B 448 (1999) 290, Phys. Rev. C61, 014901 (2000).

[17] L. Gribov, E. Levin and M. Ryskin, Phys. Rep. 100 (1983) 1.

[18] A. H. Muller and J. Qui, Nucl. Phys. B268 (1986) 427.

[19] K. J. Eskola, J. Qui and X. N. Wang, Phys. Rev. Lett. 72 (1994) 36.

[20] N. Hammon, L. Gerland, H. Stöcker and W. Greiner, Phys. Rev. C59 (1999) 2744.

[21] P. L. McGaughey, E. Quack, P. V. Ruuskanen, R. Vogt and X.-N. Wang, Int. J. Mod. Phys. A 10 (1995) 2999.

[22] John Babcock, Dennis Sivers and Stephen Wolfram, Phys. Rev. D 18 (1978) 162.

[23] B.L. Combridge, Nucl. Phys. B151 (1979) 429.

[24] M. Glueck, E. Reya and A. Vogt, Euro. Phys. J. C 5 (1998) 461.

[25] K. J. Eskola, K. Kajantie and J. Lindfors, Nucl. Phys. B 323 (1989) 37. 


\section{FIGURE CAPTIONS}

Fig. 1. The cross section for heavy-quark production via the parton fusion process $g g \rightarrow Q \bar{Q}$ at RHIC. The solid (dotted) line represents the heavy-quark production cross section (in $m b$ ) with (without) gluon shadowing (explained in the text). The dashed line represents the ratio of the cross sections with and without shadowing.

Fig. 2. The cross section for the heavy-quark production via the parton fusion process $g g \rightarrow Q \bar{Q}$ at LHC. The solid (dotted) line represents the heavy-quark production cross section (in $m b$ ) with (without) gluon shadowing (explained in the text). The dashed line represents the ratio of the cross sections with and without shadowing. It can be seen that there is a strong shadowing effect on charm production (about a factor of 10, (see the ratio curve and discussion in the text)).

Fig. 3. The cross section in $m b$ from the process $g g \rightarrow C \bar{C}$. Solid (dotted) line is with (without) gluon shadowing (explained in the text). $M_{C}=1.5 \mathrm{GeV}$ is used in the calculation.

Fig. 4. The cross section in $m b$ from the process $g g \rightarrow B \bar{B}$. Solid (dotted) line is with (without) gluon shadowing (explained in the text). $M_{B}=5.4 \mathrm{GeV}$ is used in the calculation. 


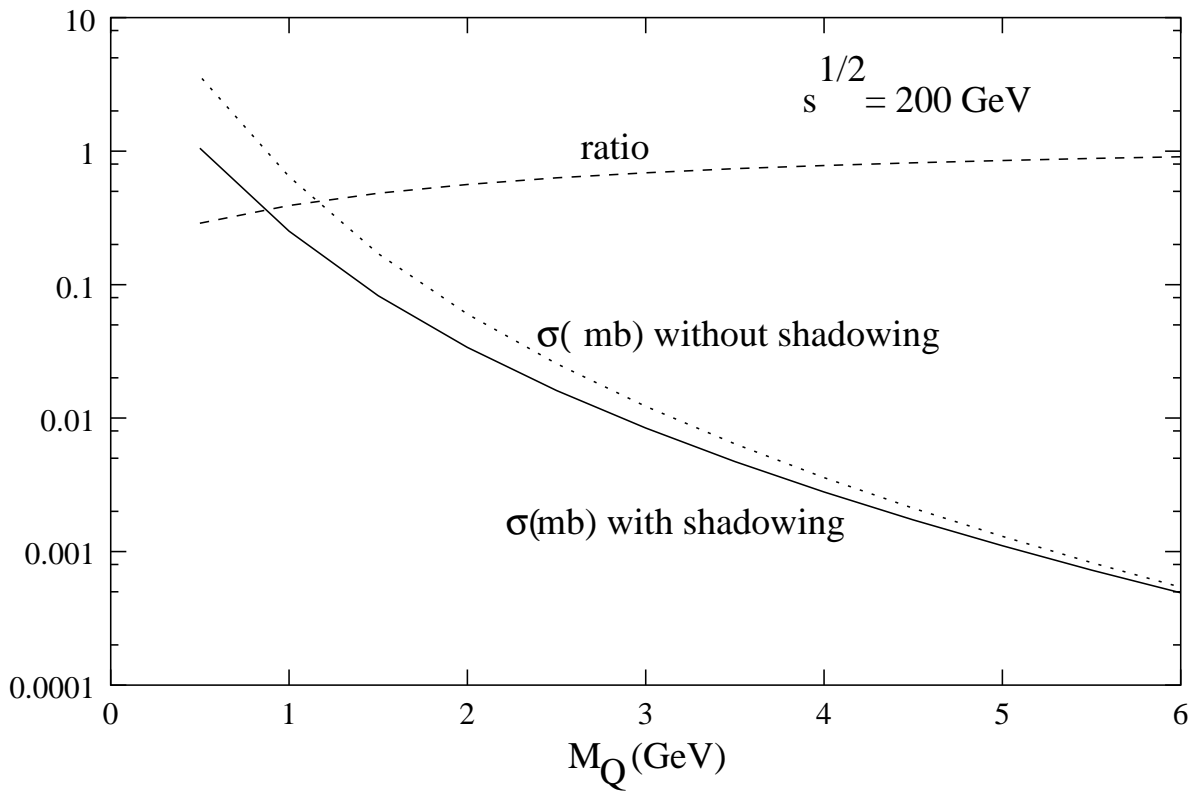




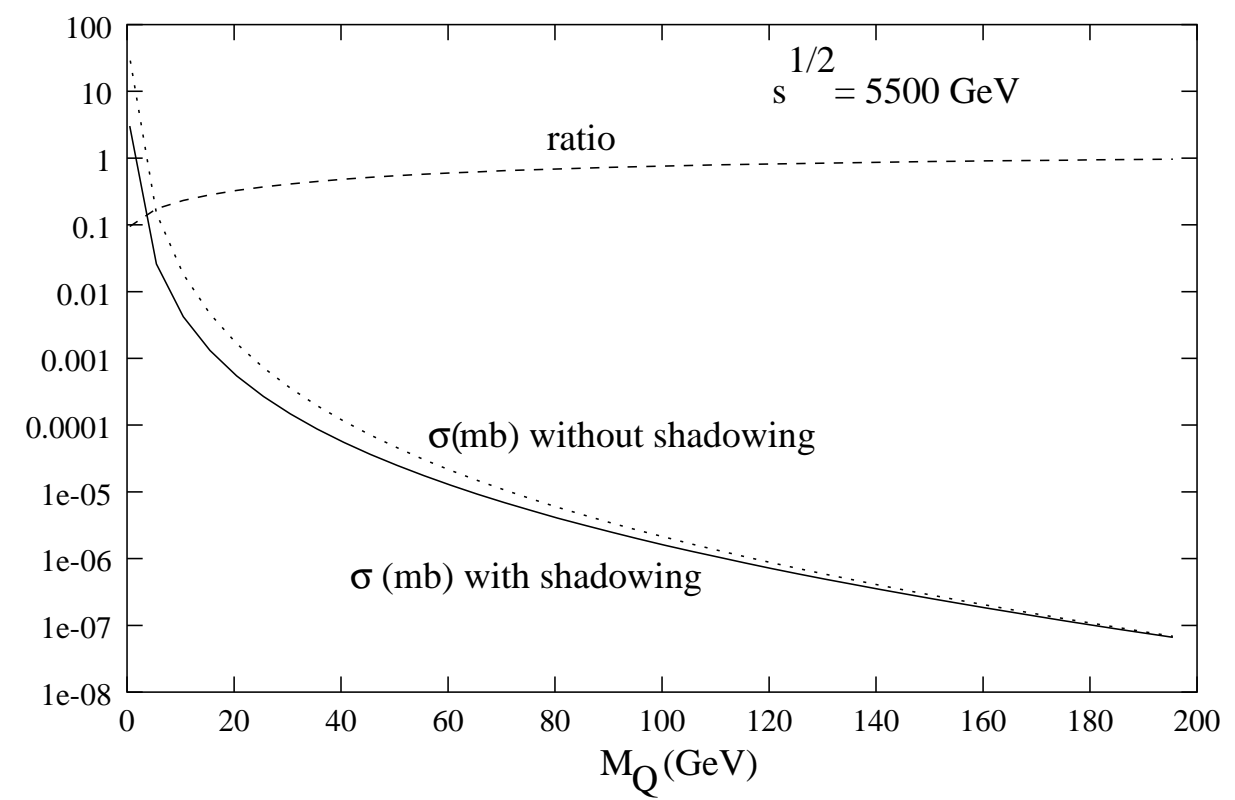




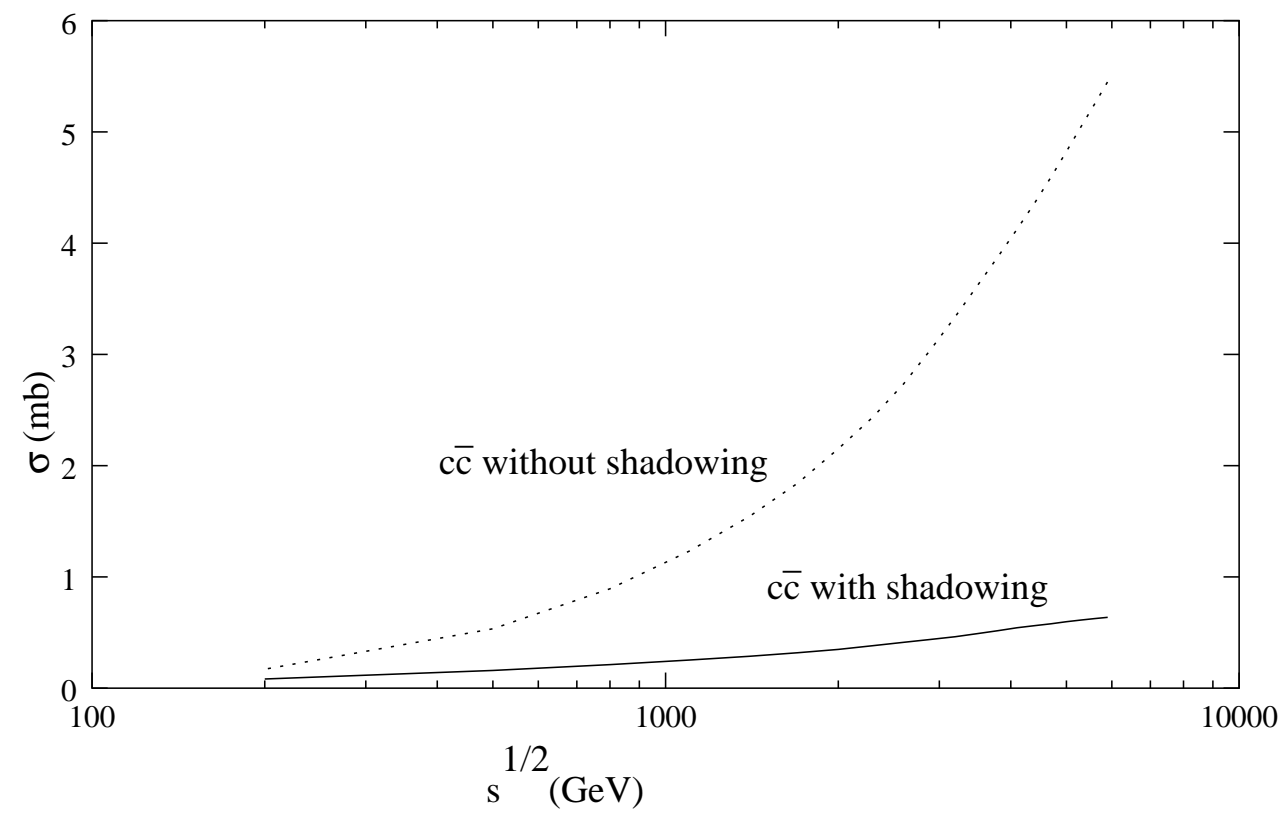




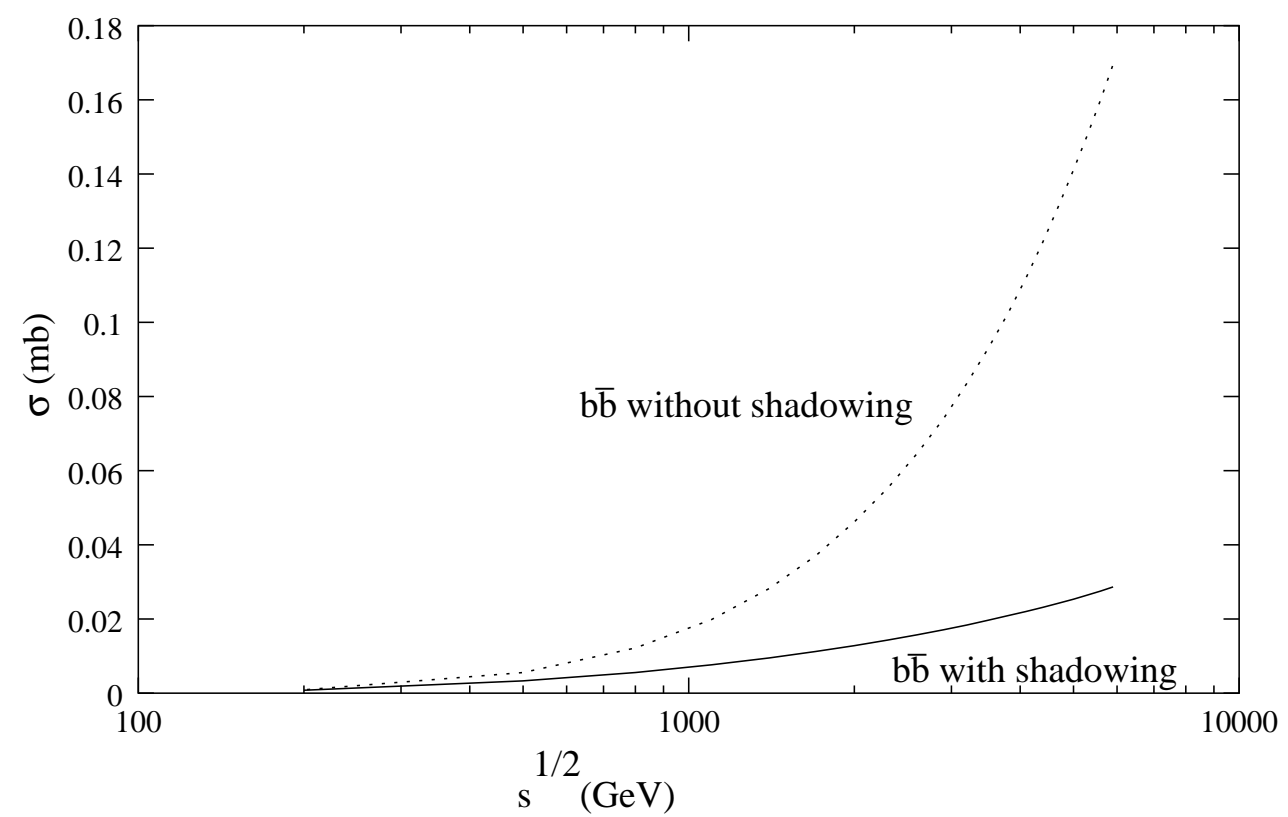

